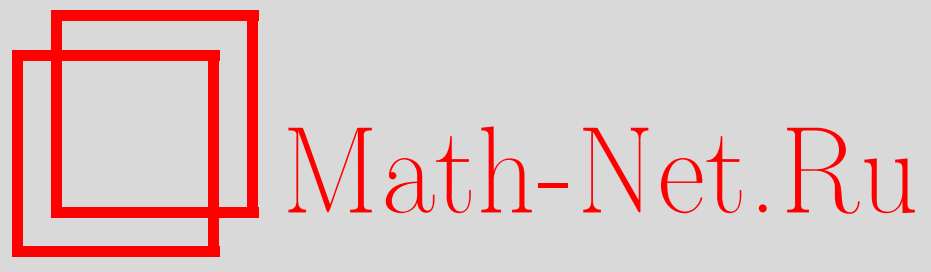

И. В. Микитюк, А. М. Степин, Достаточное условие поэтапной редукции: доказательство и приложения, $M a$ тем. сб., 2008, том 199, номер 5, 35-44

DOI: https://doi.org/10.4213/sm4504

Использование Общероссийского математического портала Math-Net.Ru подразумевает, что вы прочитали и согласны с пользовательским соглашением http://www . mathnet.ru/rus/agreement

Параметры загрузки:

IP : 3.91 .87 .62

26 апреля 2023 г., 16:40:47

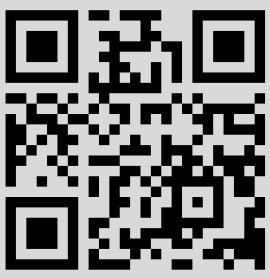




\author{
И. В. Микитюк, А. М. Степин
}

\title{
Достаточное условие поэтапной редукции: доказательство и приложения
}

\begin{abstract}
В статье доказано предположение "Stages Hypothesis" Марсдена-Миселэка-Ортеги-Перлмуттера-Ратью, которое является достаточным условием для общей теоремы о поэтапной редукции. Наш Ли-алгебраический подход позволил нам доказать это предположение в общем случае (не только для полупрямых произведений групп с коциклом) и упростить доказательство общей теоремы о поэтапной редукции (с некоторыми изменениями).

Библиография: 8 названий.
\end{abstract}

\section{Введение}

Симплектическая редукция может быть описана следующим образом: исходя из симплектического действия группы Ли на симплектическом многообразии, имеющего отображение момента, можно факторизовать поверхность уровня отображения момента по действию подходящей подгруппы, чтобы образовать новое симплектическое многообразие. До этапа факторизации мы имеем многообразие, обладающее вырожденной замкнутой 2-формой. Устраняя эту вырожденность переходом к факторпространству, получаем редуцированное симплектическое многообразие (см. [1], [2]).

Проблема осуществления поэтапной редукции возникала уже в работе Дж. Марсдена и А. Вайнстейна [1] и может быть сформулирована следующим образом: нужно предложить общую конструкцию, которая позволяет осуществить повторение редукции по двум последовательным группам симметрий и результат которой был бы таким же, как при редукции по одной бо́льшей группе (см. [2]).

Одним из самых изысканных примеров поэтапной редукции является теория редукции относительно полупрямых произведений, возникшая благодаря работам В. Гийемина и Ш. Стернберга [3] и Дж. Марсдена, Т. Ратью и А. Вайнстейна [4], [5]. Эта теория есть не только проверка того, что редукция для полупрямых произведений может быть осуществлена в два этапа; также были развиты новые математические методы, среди которых определение структуры орбит коприсоединенного представления для полупрямых произведений и теория редукции для кокасательных расслоений (см. [3]). Более того, случай полупрямого произведения был тем важным, совсем не тривиальным побудительным мотивом, который был понятен и хорошо известен уже в 1990-х годах. Естественно, что были найдены обобщения, стимулированные этой теорией.

Проблема осуществления поэтапной редукции в своей финальной и наиболее общей форме была сформулирована Марсденом и Ратью (см. [2]). Они пришли

(C) И. В. Микитюк, А. М. Степин, 2008 
к выводу, что, начиная с “большой группы" $M$ с нормальной подгруппой $N$ и редуцируя сначала по $N$, а затем по некоторой факторгруппе $M_{\nu} / N_{\nu}, M_{\nu} \subset M$, $N_{\nu} \subset N$, мы получим правильную общую схему для теории поэтапной редукции. Они показали также, что в то время как соответствующий вопрос для пуассоновой редукции довольно прост, в симплектическом случае имеются трудности.

В настоящей работе доказано (см. лемму 3 и замечание 4) предположение "Stages Hypothesis" Марсдена-Миселэка-Ортеги-Перлмуттера-Ратью, которое является достаточным условием для общей теоремы о поэтапной редукции (см. [6], [2; теорема 5.2.9]). В книге [2; §§6.1-6.4] это предположение было проверено для наиболее общего класса групповых расширений $M$ группы Ли $N$, являющихся полупрямыми произведениями с коциклом. В частности, и центральные расширения, и полупрямые произведения с векторным пространством попадают в этот класс. Здесь мы даем короткое Ли-алгебраическое доказательство этого предположения в общем случае для произвольной пары $(M, N)$ группы Ли $M$ и ее нормальной, не обязательно замкнутой подгруппы $N$. Интересно, что факт того, что условие предположения зависит только от свойств группы Ли и не зависит от данных, заключенных в действии, был отмечен в $[2 ; \S 5.2]$. Наше доказательство этого предположения основано на одном замечательном факте теории орбит коприсоединенного представления: аннулятор касательного пространства к орбите (в алгебре Ли) является алгеброй Ли группы изотропии.

Более того, здесь мы решаем проблему неэквивариантности, возникающую в [2]: начиная с эквивариантного отображения момента $J$ для группы Ли $M$ и пытаясь сначала редуцировать по $N$ (чтобы получить первое редуцированное пространство), а затем по факторгруппе $M_{\nu} / N_{\nu}$ (чтобы получить второе редуцированное пространство), получаем, вообще говоря, не гамильтоново действие этой факторгруппы на первом редуцированном пространстве, т.е. соответствующее отображение момента для группы $M_{\nu} / N_{\nu}$, индуцированное $J$, не является эквивариантным. Препятствием для эквивариантности является то, что для тривиального действия группы $N_{\nu} \subset N$ и его нулевых инфинитезимальных генераторов (гамильтоновых векторных полей) на первом редуцированном пространстве соответствующие гамильтоновы функции, индуцированные $J$, являются ненулевыми константами. Как результат симплектическое действие порождает нетривиальный коцикл на $M_{\nu} / N_{\nu}$. Этот коцикл вычислен в $[2 ; \S 5.2]$ и использован для модификации обычной процедуры редукции Марсдена и Вайнстейна, чтобы осуществить редукцию с неэквивариантным отображением момента.

В настоящей работе мы модифицируем ММОПР-процедуру поэтапной редукции, чтобы разрешить эту проблему другим путем. Мы заменяем факторгруппу $M_{\nu} / N_{\nu}$ группой $M_{\nu}$, рассматривая действие $M_{\nu}$ на первом редуцированном пространстве (и помня о том, что действие подгруппы $N_{\nu} \subset M_{\nu}$ на этом пространстве тривиально). Это действие группы $M_{\nu}$ обладает естественным эквивариантным отображением момента и допускает обычную процедуру редукции Марсдена-Вайнстейна. Полученное многообразие (второе редуцированное пространство) является таким же, как и в ММОПР-процедуре поэтапной 
редукции, но доказательство общей теоремы о поэтапной редукции, основанное на этом факте, является более коротким и ясным. Более того, мы используем более слабые условия для проведения поэтапной редукции. Например, нормальная подгруппа $N \subset M$ не обязательно замкнута и первое редуцированное пространство не обязательно связно.

\section{§1. Гамильтонова редукция}

В этом параграфе мы напомним некоторые факты о гамильтоновых действиях групп Ли и процедуре редукции. Более детальное изложение материала, помещенного ниже, можно найти в [7], [8].

Пусть $G$ - группа Ли, $\mathfrak{g}$ - ее алгебра Ли, а $\mathfrak{g}^{*}$ - дуальное пространство. Предположим, что группа $G$ симплектически действует на симплектическом многообразии $(X, \omega)$ (с симплектической формой, обозначаемой через $\omega$ ). Мы обозначим через $\widehat{\xi}$ инфинитезимальный генератор (векторное поле на $X$ ), ассоциированный с элементом алгебры Ли $\xi \in \mathfrak{g}$. Отображение $J: X \rightarrow \mathfrak{g}^{*}$ называется отображением момента, если для каждого вектора $\xi \in \mathfrak{g}$ векторное поле $\widehat{\xi}$ является гамильтоновым векторным полем ассоциированной функции $J_{\xi}(x)=\langle J(x), \xi\rangle$, т.е. функция $J_{\xi}$ удовлетворяет условию $i_{\widehat{\xi}} \omega=-d J_{\xi}$.

Симплектическое действие группы $G$ на $X$ называется гамильтоновым ( $X$ есть гамильтоново $G$-пространство), если существует эквивариантное отображение момента $J$, т.е. если

$$
J(g \cdot x)=\operatorname{Ad}_{g^{-1}}^{*}(J(x)) \quad \text { для всех } g \in G, x \in X,
$$

где $g \cdot x$ обозначает результат действия $g$ на точку $x$. Из этого свойства следует, что отображение $\xi \mapsto J_{\xi}$ является гомоморфизмом из алгебры Ли $\mathfrak{g}$ в алгебру Ли $\left(C^{\infty}(X),\{\}\right)$, т.е. $\left\{J_{\xi}, J_{\eta}\right\}=J_{[\xi, \eta]}$, где $\xi, \eta \in \mathfrak{g}$. Более того, для произвольного касательного вектора $Y \in T_{x} X$ имеем

$$
\left\langle J_{* x}(Y), \xi\right\rangle=d J_{\xi}(x)(Y)=-\omega_{x}(\widehat{\xi}(x), Y) .
$$

Для произвольного подпространства $\mathfrak{a} \subset \mathfrak{g}$ (соответственно $\left.V \subset \mathfrak{g}^{*}\right)$ обозначим через $\mathfrak{a}^{\sharp} \subset \mathfrak{g}^{*}$ (соответственно $\left.V^{\sharp}\right)$ его аннулятор в $\mathfrak{g}^{*}$ (соответственно в $\left.\mathfrak{g}\right)$. Ясно, что $\left(\mathfrak{a}^{\sharp}\right)^{\sharp}=\mathfrak{a}$. Так как форма $\omega$ невырождена, то из условия (2) следует, что

$$
J_{* x}\left(T_{x} X\right)=\mathfrak{g}_{x}^{\sharp}
$$

и, следовательно, $\mathfrak{g}_{x}=\left(J_{* x}\left(T_{x} X\right)\right)^{\sharp}$, где $\mathfrak{g}_{x}=\{\xi \in \mathfrak{g}: \widehat{\xi}(x)=0\}-$ алгебра изотропии точки $x \in X$. В силу (3) точка $x \in X$ является регулярной точкой отображения момента $J$ тогда и только тогда, когда ее алгебра изотропии $\mathfrak{g}_{x}$ зануляется.

Зафиксируем ковектор $\alpha \in \mathfrak{g}^{*}$. Пусть $G_{\alpha}$ - группа изотропии элемента $\alpha$ относительно коприсоединенного действия $G$ на $\mathfrak{g}^{*}$. Обозначим через $\mathfrak{g}_{\alpha}$ ее алгебру Ли; тогда

$$
\mathfrak{g}_{\alpha}=\left\{\xi \in \mathfrak{g}: \operatorname{ad}_{\xi}^{*} \alpha=0\right\}=\{\xi \in \mathfrak{g}:\langle\alpha,[\xi, \mathfrak{g}]\rangle=0\}, \quad \mathfrak{g}_{\alpha}=\left(\operatorname{ad}_{\mathfrak{g}}^{*} \alpha\right)^{\sharp} .
$$


Другими словами, алгебра изотропии $\mathfrak{g}_{\alpha}$ является аннулятором касательного пространства $\mathrm{ad}_{\mathfrak{g}}^{*} \alpha$ орбиты коприсоединенного представления в $\mathfrak{g}^{*}$, проходящей через точку $\alpha$, и, обратно, касательное пространство $\operatorname{ad}_{\mathfrak{g}}^{*} \alpha$ является аннулятором $\mathfrak{g}_{\alpha}$.

Предположим, что $\alpha \in \mathfrak{g}^{*}$ - слабо регулярное значение функции $J$, т.е. множество $J^{-1}(\alpha) \subset X$ является (иммерсированным) подмногообразием с касательным расслоением ker $J_{*} \mid J^{-1}(\alpha)$. В силу эквивариантности $J$ подмногообразие $J^{-1}(\alpha)$ инвариантно относительно действия группы $G_{\alpha}$, так что пространство орбит $X_{\alpha}=J^{-1}(\alpha) / G_{\alpha}^{\prime}$, где $G_{\alpha}^{\prime} \subset G_{\alpha}-$ (открытая) подгруппа в $G_{\alpha}$, содержащая компоненту единицы $G_{\alpha}^{0} \subset G_{\alpha}$, корректно определено. Пусть $i_{\alpha}: J^{-1}(\alpha) \rightarrow X$ обозначает включение. Мы будем говорить, что подмногообразие $J^{-1}(\alpha)$ является $G_{\alpha}^{\prime}$-редуцируемьм, если на факторпространстве $X_{\alpha}$ существует структура многообразия такая, что каноническая проекция

$$
\pi_{\alpha}: J^{-1}(\alpha) \rightarrow X_{\alpha}
$$

является субмерсией (если такая структура существует, то она единственна).

Теорема 1 (редукция Марсдена-Вайнстейна; см. [1]). Пусть $(X, \omega)-$ гамильтоново $G$-пространство. Предположим, что $\alpha \in \mathfrak{g}^{*}$ - слабо регулярное значение отображения момента $J$ и подмногообразие $J^{-1}(\alpha)$ является $G_{\alpha}^{\prime}$-редуцируемым. Тогда на многообразии $X_{\alpha}$ существует единственная симплектическая структура $\omega_{\alpha}$ такая, что $\pi_{\alpha}^{*} \omega_{\alpha}=i_{\alpha}^{*} \omega$.

ЗАмечАниЕ 2 . Пусть $J^{1}$ - другое эквивариантное отображение момента на гамильтоновом $G$-пространстве $(X, \omega)$. Если многообразие $X$ связно, то $J^{1}=$ $J+\mu$, где $\mu \in \mathfrak{g}^{*}$ и $\operatorname{Ad}^{*}(G)(\mu)=\mu$. Поэтому для произвольных $\alpha \in \mathfrak{g}^{*}$ имеем $J^{-1}(\alpha)=\left(J^{1}\right)^{-1}(\alpha+\mu)$. Так как $G_{\alpha}=G_{\alpha+\mu}$, то редуцированное многообразие $X_{\alpha}$ не зависит от выбора отображения момента $J$.

\section{§ 2. Гамильтонова поэтапная редукция}

2.1. Алгебраические предпосылки: доказательство предположения “Stages Hypothesis". Пусть $M$ - группа Ли с нормальной подгруппой $N$ (не обязательно замкнутой и связной). Обозначим через $\mathfrak{m}$ и $\mathfrak{n}$ соответствующие алгебры Ли. Пусть $\mathrm{Ad}^{*}: M \rightarrow \operatorname{End}\left(\mathfrak{m}^{*}\right)$ - коприсоединенное представление группы Ли $M$ в дуальном пространстве $\mathfrak{m}^{*}$. Так как мы будем рассматривать также некоторые подгруппы группы $M$, то через $\mathrm{Ad}_{m}^{*}$ и $\operatorname{ad}_{\xi}^{*}$ будем обозначать только операторы в пространстве $\mathfrak{m}^{*}$, через $\mathrm{Ad}_{m}$ и $\operatorname{ad}_{\xi}$ - операторы в $\mathfrak{m}$. Зафиксируем некоторый линейный функционал $\sigma \in \mathfrak{m}^{*}$, обозначим через $M_{\sigma}$ группу изотропии элемента $\sigma$ и через $\mathfrak{m}_{\sigma}$ ее алгебру Ли. Положим $N_{\sigma}=N \cap M_{\sigma}$ и $\mathfrak{n}_{\sigma}=\mathfrak{n} \cap \mathfrak{m}_{\sigma}$. Подгруппа $N_{\sigma}$ является замкнутой подгруппой в $N$ с алгеброй Ли $\mathfrak{n}_{\sigma}$. По определению

$$
\mathfrak{m}_{\sigma}=\{\xi \in \mathfrak{m}:\langle\sigma,[\xi, \mathfrak{m}]\rangle=0\}, \quad \mathfrak{n}_{\sigma}=\{y \in \mathfrak{n}:\langle\sigma,[y, \mathfrak{m}]\rangle=0\} .
$$

Поскольку подалгебра $\mathfrak{n}$ является идеалом в $\mathfrak{m}$, то присоединенное представление $\mathfrak{m}$ индуцирует представление $\rho$ алгебры $\mathfrak{m}$ в $\mathfrak{n}$, а присоединенное действие $\operatorname{Ad}: M \rightarrow \operatorname{End}(\mathfrak{m})$ группы $M$ индуцирует $M$-действие на $\mathfrak{n}: M \times \mathfrak{n} \rightarrow \mathfrak{n}$, 
$(m, \xi) \mapsto \operatorname{Ad}_{m} \xi$. Для дуального представления $\rho^{*}$ алгебры $\mathfrak{m}$ в $\mathfrak{n}^{*}$ имеем

$$
\left\langle\rho_{\xi}^{*} \mu, y\right\rangle=\langle\mu,[\xi, y]\rangle, \quad \text { где } \xi \in \mathfrak{m}, \mu \in \mathfrak{n}^{*}, y \in \mathfrak{n} .
$$

Соответствующее $M$-действие на $\mathfrak{n}^{*}$ определяется уравнением $\langle m \cdot \mu, y\rangle=$ $\left\langle\mu, \operatorname{Ad}_{m} y\right\rangle$. Ограничение этого действия на подгруппу $N$ является ее коприсоединенным действием. Более того, каноническая проекция

$$
\pi_{\mathfrak{n}^{*}}: \mathfrak{m}^{*} \rightarrow \mathfrak{n}^{*}, \quad \alpha \mapsto \alpha \mid \mathfrak{n},
$$

является $M$-эквивариантным отображением относительно этих двух действий $M$ на пространствах $\mathfrak{m}^{*}$ и $\mathfrak{n}^{*}$ соответственно [2; § 5.2]:

$$
\pi_{\mathfrak{n}^{*}}\left(\operatorname{Ad}_{m}^{*} \alpha\right)=m \cdot \pi_{\mathfrak{n}^{*}}(\alpha) \quad \text { для всех } \alpha \in \mathfrak{m}^{*}, m \in M .
$$

В самом деле, для произвольных $y \in \mathfrak{n}$

$$
\left\langle\pi_{\mathfrak{n}^{*}}\left(\operatorname{Ad}_{m}^{*} \alpha\right), y\right\rangle=\left\langle\operatorname{Ad}_{m}^{*} \alpha, y\right\rangle=\left\langle\alpha, \operatorname{Ad}_{m} y\right\rangle=\left\langle\pi_{\mathfrak{n}^{*}}(\alpha), \operatorname{Ad}_{m} y\right\rangle=\left\langle m \cdot \pi_{\mathfrak{n}^{*}}(\alpha), y\right\rangle .
$$

Рассмотрим ограничение $\nu=\pi_{\mathfrak{n}}(\sigma) \in \mathfrak{n}^{*}$ элемента $\sigma \in \mathfrak{m}^{*}$. Обозначим через $M_{\nu}$ и $N_{\nu}$ группы изотропии элемента $\nu$, а через $\mathfrak{m}_{\nu}$ и $\mathfrak{n}_{\nu}$ соответствующие алгебры Ли. Ясно, что $\mathfrak{n}_{\nu}=\mathfrak{n} \cap \mathfrak{m}_{\nu}$ и подгруппа $N_{\nu}=N \cap M_{\nu}$ является нормальной подгруппой в $M_{\nu}$. Так как $[\mathfrak{m}, \mathfrak{n}] \subset \mathfrak{n}$, то в силу определения

$$
\begin{aligned}
\mathfrak{m}_{\nu} & =\left\{\xi \in \mathfrak{m}: \rho_{\xi}^{*} \nu=0\right\}=\{\xi \in \mathfrak{m}:\langle\nu,[\xi, \mathfrak{n}]\rangle=0\}, \\
M_{\nu} & =\{m \in M: m \cdot \nu=\nu\}=\left\{m \in M: \operatorname{Ad}_{m}^{*}\left(\sigma+\mathfrak{n}^{\sharp}\right)=\sigma+\mathfrak{n}^{\sharp}\right\}, \\
\mathfrak{n}_{\nu} & =\{y \in \mathfrak{n}:\langle\nu,[y, \mathfrak{n}]\rangle=0\}=\left\{y \in \mathfrak{n}: \rho_{y^{\prime}}^{*} \nu(y)=0 \forall y^{\prime} \in \mathfrak{n}\right\} .
\end{aligned}
$$

Аналогично, в силу соотношения $[\mathfrak{m}, \mathfrak{n}] \subset \mathfrak{n}$ алгебра $\mathfrak{n}_{\sigma}$ зависит только от ограничения $\sigma \mid \mathfrak{n}=\nu$ :

$$
\mathfrak{n}_{\sigma}=\{y \in \mathfrak{n}:\langle\nu,[y, \mathfrak{m}]\rangle=0\}=\left\{y \in \mathfrak{n}: \rho_{\xi}^{*} \nu(y)=0 \forall \xi \in \mathfrak{m}\right\} ;
$$

или, что эквивалентно,

$$
\mathfrak{n}_{\sigma^{\prime}}=\mathfrak{n}_{\sigma}, \quad \text { если } \sigma^{\prime}|\mathfrak{n}=\sigma| \mathfrak{n}, \quad \text { где } \sigma^{\prime} \in \mathfrak{m}^{*} .
$$

Из соотношений (7) и (8) вытекает, что алгебры $\mathfrak{n}_{\nu}$ и $\mathfrak{n}_{\sigma}$ являются аннуляторами подпространств $\rho_{\mathfrak{n}}^{*} \nu \subset \mathfrak{n}^{*}$ и $\rho_{\mathfrak{m}}^{*} \nu \subset \mathfrak{n}^{*}$ в пространстве $\mathfrak{n}$. Однако ковектор $\rho_{\xi}^{*} \nu$ с $\xi \in \mathfrak{m}$ есть элемент подпространства $\rho_{\mathfrak{n}}^{*} \nu$ тогда и только тогда, когда $\xi \in \mathfrak{n}+\mathfrak{m}_{\nu} ;$ поэтому

$$
\operatorname{dim}\left(\mathfrak{n}_{\nu} / \mathfrak{n}_{\sigma}\right)=\operatorname{dim}\left(\rho_{\mathfrak{m}}^{*} \nu / \rho_{\mathfrak{n}}^{*} \nu\right)=\operatorname{dim}\left(\mathfrak{n}+\mathfrak{m}_{\nu}\right)^{\sharp} .
$$

Пусть $\mathfrak{n}_{\nu}^{\prime} \subset \mathfrak{n}_{\nu}-$ ядро ограничения $\nu \mid \mathfrak{n}_{\nu}$, т.е. $\mathfrak{n}_{\nu}^{\prime}=\operatorname{ker} \nu \cap \mathfrak{n}_{\nu}$. Отметим, что $\mathfrak{n}_{\nu}^{\prime}=\mathfrak{n}_{\nu}$, или $\operatorname{dim}\left(\mathfrak{n}_{\nu} / \mathfrak{n}_{\nu}^{\prime}\right)=1$. В силу $(5)$

$$
\left[\mathfrak{m}_{\nu}, \mathfrak{n}\right] \subset \operatorname{ker} \nu, \quad\left[\mathfrak{m}_{\nu}, \mathfrak{n}_{\nu}\right] \subset\left(\mathfrak{m}_{\nu} \cap \mathfrak{n}\right) \cap \operatorname{ker} \nu=\mathfrak{n}_{\nu}^{\prime},
$$

а значит, подпространство $\mathfrak{n}_{\nu}^{\prime}-$ идеал в $\mathfrak{m}_{\nu}$. Более того, так как $n \cdot \nu=\nu$ и по определению $\langle n \cdot \nu, y\rangle=\left\langle\nu, \operatorname{Ad}_{n} y\right\rangle, y \in \mathfrak{n}$, то

$$
\operatorname{Ad}_{n}\left(\mathfrak{n}_{\nu}^{\prime}\right)=\mathfrak{n}_{\nu}^{\prime} \quad \text { для всех } n \in N_{\nu} .
$$


Теперь рассмотрим представление $n \mapsto \operatorname{Ad}_{n} \mid \mathfrak{m}_{\nu}$ группы Ли $N_{\nu} \subset M_{\nu}$. Это представление индуцирует тривиальное представление компоненты единицы $N_{\nu}^{0} \subset N_{\nu}$ в факторалгебре $\mathfrak{m}_{\nu} / \mathfrak{n}_{\nu}^{\prime}$, поскольку $\left[\mathfrak{n}_{\nu}, \mathfrak{m}_{\nu}\right] \subset \mathfrak{n}_{\nu}^{\prime}$ (соответствующий гомоморфизм алгебр Ли тривиален). Таким образом (см. [2; §5.2]),

$$
\operatorname{Ad}_{n} \xi-\xi \in \mathfrak{n}_{\nu}^{\prime} \quad \text { для всех } n \in N_{\nu}^{0}, \xi \in \mathfrak{m}_{\nu} .
$$

Обозначим через $\tau$ ограничение $\sigma \mid \mathfrak{m}_{\nu}$ элемента $\sigma \in \mathfrak{m}^{*}$. Используя пару ковекторов $\nu \in \mathfrak{n}^{*}$ и $\tau \in \mathfrak{m}_{\nu}^{*}$, определим аффинное подпространство $\mathscr{A}_{\nu, \tau} \subset \mathfrak{m}^{*}$ следующим образом:

$$
\mathscr{A}_{\nu, \tau}=\left\{\alpha \in \mathfrak{m}^{*}: \alpha|\mathfrak{n}=\nu, \alpha| \mathfrak{m}_{\nu}=\tau\right\}=\sigma+\left(\mathfrak{n}+\mathfrak{m}_{\nu}\right)^{\sharp} .
$$

Мы утверждаем, что это аффинное подпространство $\mathscr{A}_{\nu, \tau} \subset \mathfrak{m}^{*}$ инвариантно относительно действия связной группы $N_{\nu}^{0} \subset M$ и, более того, что $N_{\nu}^{0}$ действует транзитивно на $\mathscr{A}_{\nu, \tau}$.

В самом деле, пусть $\alpha \in \mathscr{A}_{\nu, \tau}$ и $n \in N_{\nu}^{0}$. Так как $N_{\nu}^{0} \subset N \cap M_{\nu}$ и $\mathscr{A}_{\nu, \tau} \subset$ $\sigma+\mathfrak{n}^{\sharp}$, то в силу (6) $\operatorname{Ad}_{n}^{*} \alpha \mid \mathfrak{n}=\nu$. Для доказательства того, что $\operatorname{Ad}_{n}^{*} \alpha \mid \mathfrak{m}_{\nu}=\tau$, отметим, что в силу (13) для всех векторов $\xi \in \mathfrak{m}_{\nu}$ имеем

$$
\left\langle\operatorname{Ad}_{n}^{*} \alpha-\alpha, \xi\right\rangle=\left\langle\alpha, \operatorname{Ad}_{n} \xi-\xi\right\rangle \in\left\langle\alpha, \mathfrak{n}_{\nu}^{\prime}\right\rangle=\left\langle\nu, \mathfrak{n}_{\nu}^{\prime}\right\rangle=0
$$

т.е.

$$
\operatorname{Ad}^{*}\left(N_{\nu}^{0}\right)\left(\mathscr{A}_{\nu, \tau}\right) \subset \mathscr{A}_{\nu, \tau}
$$

Как показано выше, алгебра Ли $\mathfrak{n}_{\sigma}$ определяется только ограничением $\sigma \mid \mathfrak{n}=\nu$, и поэтому $\mathfrak{n}_{\sigma}=\mathfrak{n}_{\alpha}$ (см. (9)). Учитывая условие (10), получим, что $\operatorname{Ad}^{*}\left(N_{\nu}^{0}\right)$-орбита в пространстве $\mathscr{A}_{\nu, \tau}$, проходящая через элемент $\alpha$ (изоморфная факторпространству $\left.N_{\nu}^{0} /\left(N_{\alpha} \cap N_{\nu}^{0}\right)\right)$, является открытым подмножеством B $\mathscr{A}_{\nu, \tau}$ :

$$
\operatorname{dim} N_{\nu}^{0} /\left(N_{\alpha} \cap N_{\nu}^{0}\right)=\operatorname{dim} N_{\nu}^{0} /\left(N_{\sigma} \cap N_{\nu}^{0}\right)=\operatorname{dim}\left(\mathfrak{n}+\mathfrak{m}_{\nu}\right)^{\sharp}=\operatorname{dim} \mathscr{A}_{\nu, \tau} .
$$

Так как пространство $\mathscr{A}_{\nu, \tau}$ связно, то эта орбита совпадает со всем пространством $\mathscr{A}_{\nu, \tau}$, т.е. $\operatorname{Ad}^{*}\left(N_{\nu}^{0}\right)$ действует транзитивно на $\mathscr{A}_{\nu, \tau} ;$ так как дополнительно пространство $\mathscr{A}_{\nu, \tau}$ односвязно, то группа изотропии $N_{\alpha} \cap N_{\nu}^{0}$ связна, т.е. равна $N_{\alpha}^{0}$ (компоненте единицы группы $N_{\alpha} \subset N_{\nu}$ ).

Но группа $N_{\nu}$ является подгруппой группы изотропии $M_{\nu}$ с алгеброй Ли $\mathfrak{m}_{\nu}$. Обозначим через $\left(M_{\nu}\right)_{\tau}$ подгруппу изотропии элемента $\tau=\sigma \mid \mathfrak{m}_{\nu}$ относительно естественного коприсоединенного действия $M_{\nu}$ на $\mathfrak{m}_{\nu}^{*}$. Учитывая, что это действие определяется Ad-действием группы $M$ на $\mathfrak{m}$, из соотношения (14) получаем, что группа $\left(M_{\nu}\right)_{\tau} \cap N_{\nu}$ содержит компоненту единицы $N_{\nu}^{0}$ группы $N_{\nu}$. Отметим также, что по определению

$$
M_{\sigma} \subset M_{\nu}, \quad M_{\sigma} \subset\left(M_{\nu}\right)_{\tau} .
$$

Следующая лемма суммирует уже доказанные результаты.

Лемма 3. Для произвольного $\sigma \in \mathfrak{m}^{*}$

$$
\operatorname{Ad}^{*}\left(N_{\nu}^{0}\right) \sigma=\sigma+\left(\mathfrak{n}+\mathfrak{m}_{\nu}\right)^{\sharp}, \quad N_{\nu}^{0} \subset\left(M_{\nu}\right)_{\tau},
$$

əде $\nu=\sigma|\mathfrak{n} u \tau=\sigma| \mathfrak{m}_{\nu}$. 
ЗАмЕчАНИЕ 4 . В силу леммы 3 для всех элементов $\sigma_{1}$ и $\sigma_{2}$ из $\mathfrak{m}^{*}$ таких, что

$$
\sigma_{1}\left|\mathfrak{n}=\sigma_{2}\right| \mathfrak{n}=\nu, \quad \sigma_{1}\left|\mathfrak{m}_{\nu}=\sigma_{2}\right| \mathfrak{m}_{\nu}=\tau
$$

(т.е. $\left.\sigma_{1}, \sigma_{2} \in \mathscr{A}_{\nu, \tau}=\sigma+\left(\mathfrak{n}+\mathfrak{m}_{\nu}\right)^{\sharp}\right)$, существует элемент $n \in N_{\nu}^{0}$ такой, что $\operatorname{Ad}_{n}^{*}\left(\sigma_{1}\right)=\sigma_{2}$. В таком виде утверждение леммы 3 сформулировано в статье [6] как предположение "Stages Hypothesis", а в более слабой версии - в книге [2], где требуется, чтобы $n \in\left(M_{\nu}\right)_{\tau} \supset N_{\nu}^{0}$.

2.2. Новое доказательство теоремы о поэтапной редукции. Мы используем обозначения из п. 2.1. Пусть $(P, \omega)$ обозначает симплектическое многообразие, а $M$ - группу Ли (не обязательно связную), действующую на $P$ гамильтоновым образом с отображением момента

$$
J: P \rightarrow \mathfrak{m}^{*} .
$$

Положим $J_{\xi}(p)=\langle J(p), \xi\rangle$ для всех $p \in P$ и $\xi \in \mathfrak{m}$. Ясно, что действие нормальной подгруппы $N \subset M$ на $P$ также гамильтоново (как ограничение гамильтонова действия группы $M$ ) с отображением момента

$$
J^{\prime}: P \rightarrow \mathfrak{n}^{*}, \quad \text { где } J^{\prime}(p)=J(p) \mid \mathfrak{n} \quad \text { для всех } p \in P .
$$

Тогда для функции $J_{y}^{\prime}(p) \stackrel{\text { def }}{=}\left\langle J^{\prime}(p), y\right\rangle$, где $p \in P$ и $y \in \mathfrak{n}$, имеем $J_{y}^{\prime}=J_{y}$.

Предположим, что $\sigma \in \mathfrak{m}^{*}$ и $\sigma \mid \mathfrak{n}=\nu \in \mathfrak{n}^{*}$ являются слабо регулярными значениями отображений моментов $J$ и $J^{\prime}$ соответственно. Предположим также, что пространство $J^{-1}(\sigma) M_{\sigma}$-редуцируемо, а $\left(J^{\prime}\right)^{-1}(\nu) N_{\nu}^{0}$-редуцируемо. Тогда в силу теоремы Марсдена-Вайнстейна о редукции редуцированные пространства $P_{\sigma}=J^{-1}(\sigma) / M_{\sigma}$ и $P_{\nu}^{\prime}=\left(J^{\prime}\right)^{-1}(\nu) / N_{\nu}^{0}$ являются симплектическими многообразиями с включениями

$$
i_{\sigma}: J^{-1}(\sigma) \rightarrow P, \quad i_{\nu}^{\prime}:\left(J^{\prime}\right)^{-1}(\nu) \rightarrow P,
$$

каноническими проекциями

$$
\pi_{\sigma}: J^{-1}(\sigma) \rightarrow P_{\sigma}, \quad \pi_{\nu}^{\prime}:\left(J^{\prime}\right)^{-1}(\nu) \rightarrow P_{\nu}^{\prime}
$$

и симплектическими формами $\omega_{\sigma}$ и $\omega_{\nu}^{\prime}$ (на $P_{\sigma}$ и $P_{\nu}^{\prime}$ соответственно).

Пусть $M_{\nu}$ обозначает (как и в п. 2.1) группу изотропии элемента $\nu$ для действия $M$ на $\mathfrak{n}^{*}$. Эта подгруппа имеет важное значение для дальнейшего изложения.

Так как по определению $\left(J^{\prime}\right)^{-1}(\nu)=J^{-1}\left(\sigma+\mathfrak{n}^{\sharp}\right)$ и аффинное подпространство $\sigma+\mathfrak{n}^{\sharp} \operatorname{Ad}^{*}\left(M_{\nu}\right)$-инвариантно, то в силу (6) и в силу эквивариантности (1) отображения момента $J$ получим, что подмногообразие $\left(J^{\prime}\right)^{-1}(\nu) \subset P M_{\nu}$-инвариантно. Более того, это действие $M_{\nu}$ определяет единственное $M_{\nu}$-действие на факторпространстве $P_{\nu}^{\prime}$ такое, что диаграмма

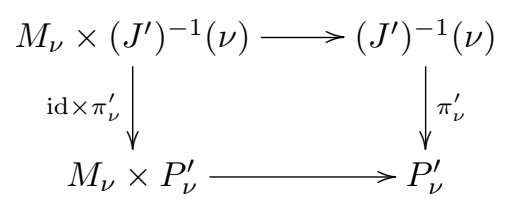


коммутативна, потому что подгруппа $N_{\nu} \subset M_{\nu}$ и, следовательно, ее компонента единицы $N_{\nu}^{0}$ является нормальной подгруппой в $M_{\nu}$. Это действие группы $M_{\nu}$ является гладким.

Более того, ограничение (функция) $J_{\xi} \mid\left(J^{\prime}\right)^{-1}(\nu)$ с $\xi \in \mathfrak{m}_{\nu} N_{\nu}^{0}$-инвариантно. В самом деле, для произвольной точки $p \in\left(J^{\prime}\right)^{-1}(\nu)=J^{-1}\left(\sigma+\mathfrak{n}^{\sharp}\right)$ и $n \in N_{\nu}^{0}$

$$
J_{\xi}\left(n^{-1} \cdot p\right)-J_{\xi}(p)=J_{\operatorname{Ad}_{n} \xi}(p)-J_{\xi}(p) \in\left\langle\sigma+\mathfrak{n}^{\sharp}, \operatorname{Ad}_{n} \xi-\xi\right\rangle=\left\langle\nu, \operatorname{Ad}_{n} \xi-\xi\right\rangle=0,
$$

потому что вектор $\operatorname{Ad}_{n} \xi-\xi$ - элемент ядра $\mathfrak{n}_{\nu}^{\prime}=\operatorname{ker}\left(\nu \mid \mathfrak{n}_{\nu}\right) \subset \mathfrak{n}_{\nu}($ см. (13)). Таким образом, существует единственное корректно определенное отображение $J^{\prime \prime}$ на факторпространстве $P_{\nu}^{\prime}=\left(J^{\prime}\right)^{-1}(\nu) / N_{\nu}^{0}$

$$
J^{\prime \prime}: P_{\nu}^{\prime} \rightarrow \mathfrak{m}_{\nu}^{*}
$$

такое, что

$$
\left\langle\left(J^{\prime \prime} \circ \pi_{\nu}^{\prime}\right)(p), \xi\right\rangle=\langle J(p), \xi\rangle \quad \text { для всех } p \in\left(J^{\prime}\right)^{-1}(\nu), \xi \in \mathfrak{m}_{\nu} .
$$

Это отображение гладко, потому что $\pi_{\nu}^{\prime}-$ субмерсия и диаграмма

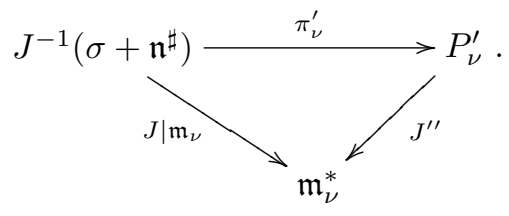

коммутативна.

Следующая теорема является некоторым обобщением теоремы о поэтапной редукции из [2].

ТЕОрема 5. Предположим, что $\sigma \in \mathfrak{m}^{*} u \sigma \mid \mathfrak{n}=\nu \in \mathfrak{n}^{*}-$ слабо регулярные значения отображений моментов $J$ и $J^{\prime}$ соответственно. Допустим также, что пространство $J^{-1}(\sigma) M_{\sigma}$-редуиируемо, а $\left(J^{\prime}\right)^{-1}(\nu) N_{\nu}^{0}$-редуиируемо. Тогда действие $M$ на $P$ индуцирует гамильтоново действие группъ Ли $M_{\nu} н а$ симплектическом многообразии $\left(P_{\nu}^{\prime}, \omega_{\nu}^{\prime}\right)$ с отображением момента $J^{\prime \prime}: P_{\nu}^{\prime} \rightarrow$ $\mathfrak{m}_{\nu}^{*}($ см. (17)) - единственным отображением, для которого выполнено условие (18).

Предположим, что ковектор $\tau=\sigma \mid \mathfrak{m}_{\nu} \in \mathfrak{m}_{\nu}^{*}$ является слабо регулярным значением отображения момента $J^{\prime \prime}$ и что пространство $\left(J^{\prime \prime}\right)^{-1}(\tau)$ $\left(M_{\nu}\right)_{\tau}$-редуцируемо. Тогда симплектические многообразия (редуиированные пространства) $\left(P_{\nu \tau}^{\prime \prime}, \omega_{\nu \tau}^{\prime \prime}\right)$ u $\left(P_{\sigma}, \omega_{\sigma}\right)$ симплектически диффеоморфны. Здесъ $\left(P_{\nu \tau}^{\prime \prime}, \omega_{\nu \tau}^{\prime \prime}\right)$ обозначает факторпространство $P_{\nu \tau}^{\prime \prime}=\left(J^{\prime \prime}\right)^{-1}(\tau) /\left(M_{\nu}\right)_{\tau}$ c единственной симплектической формой $\omega_{\nu \tau}^{\prime \prime}$ такой, что $\left(\pi_{\nu \tau}^{\prime \prime}\right)^{*} \omega_{\nu \tau}^{\prime \prime}=\left(i_{\nu \tau}^{\prime \prime}\right)^{*} \omega_{\nu}^{\prime}$, где

$$
i_{\nu \tau}^{\prime \prime}:\left(J^{\prime \prime}\right)^{-1}(\tau) \rightarrow P_{\nu}^{\prime}, \quad \pi_{\nu \tau}^{\prime \prime}:\left(J^{\prime \prime}\right)^{-1}(\tau) \rightarrow P_{\nu \tau}^{\prime \prime}
$$

являются включением и канонической проекцией соответственно.

ДокАЗАТЕЛЬСтво. Пусть $p \in\left(J^{\prime}\right)^{-1}(\nu)$ и $Y$ - вектор, касательный к подмногообразию $\left(J^{\prime}\right)^{-1}(\nu)$ в $p$. Пусть $\xi \in \mathfrak{m}_{\nu}$ и $m \in M_{\nu}$. Нужно доказать, что отображение $J^{\prime \prime}$ является (эквивариантным) отображением момента для $M_{\nu}$-действия 
на $P_{\nu}^{\prime}$. Этот факт следует из определений отображения $J^{\prime \prime}($ см. (18)) и симплектической формы $\omega_{\nu}^{\prime}$ :

$$
d J_{\xi}^{\prime \prime}\left(\pi^{\prime}{ }_{\nu *} Y\right)=d J_{\xi}(Y)=-\omega(\widehat{\xi}, Y)=-\omega_{\nu}^{\prime}\left(\pi^{\prime}{ }_{\nu *} \widehat{\xi}, \pi^{\prime}{ }_{\nu *} Y\right),
$$

и из определения действия $M_{\nu}(16)$, а также эквивариантности отображения $J$ :

$$
\begin{aligned}
\left\langle J^{\prime \prime}\left(m \cdot \pi_{\nu}^{\prime}(p)\right), \xi\right\rangle & =\left\langle J^{\prime \prime}\left(\pi_{\nu}^{\prime}(m \cdot p)\right), \xi\right\rangle=\langle J(m \cdot p), \xi\rangle \\
& =\left\langle J(p), \operatorname{Ad}_{m^{-1}} \xi\right\rangle=\left\langle J^{\prime \prime}\left(\pi_{\nu}^{\prime}(p)\right), \operatorname{Ad}_{m^{-1}} \xi\right\rangle .
\end{aligned}
$$

Чтобы сконструировать диффеоморфизм $\phi: P_{\nu \tau}^{\prime \prime} \rightarrow P_{\sigma}$, отметим, что по определению (18)

$$
\left(J^{\prime \prime}\right)^{-1}(\tau)=\pi_{\nu}^{\prime}\left(J^{-1}\left(\sigma+\left(\mathfrak{n}+\mathfrak{m}_{\nu}\right)^{\sharp}\right)\right) .
$$

Множество в правой части равенства корректно определено, поскольку аффинное пространство $\sigma+\left(\mathfrak{n}+\mathfrak{m}_{\nu}\right)^{\sharp}$ является подпространством в $\sigma+\mathfrak{n}^{\sharp}$, так что

$$
J^{-1}\left(\sigma+\left(\mathfrak{n}+\mathfrak{m}_{\nu}\right)^{\sharp}\right) \subset J^{-1}\left(\sigma+\mathfrak{n}^{\sharp}\right)=\left(J^{\prime}\right)^{-1}(\nu) .
$$

Поэтому чтобы сконструировать $\phi$, достаточно установить взаимно однозначное соответствие между множеством всех $\left(M_{\nu}\right)_{\tau}$-орбит в множестве $J^{-1}(\sigma+$ $\left.\left(\mathfrak{n}+\mathfrak{m}_{\nu}\right)^{\sharp}\right)$ (в силу леммы $3 N_{\nu}^{0} \subset\left(M_{\nu}\right)_{\tau}$, т.е. $\left.\left(M_{\nu}\right)_{\tau} \cdot N_{\nu}^{0}=\left(M_{\nu}\right)_{\tau}\right)$ и множеством всех $M_{\sigma}$-орбит в множестве $J^{-1}(\sigma)$. В самом деле, используя эквивариантность отображения момента $J$ и лемму 3 , получим, что каждая $\left(M_{\nu}\right)_{\tau}$-орбита в множестве $J^{-1}\left(\sigma+\left(\mathfrak{n}+\mathfrak{m}_{\nu}\right)^{\sharp}\right)$ пересекает множество $J^{-1}(\sigma)$. Это пересечение является $M_{\sigma}$-орбитой в $J^{-1}(\sigma)$, что вытекает из повторного использования эквивариантности отображения момента:

$$
J\left(m^{-1} \cdot p\right)=\sigma \quad \text { для } p \in J^{-1}(\sigma), m \in M \quad \Longleftrightarrow \quad \operatorname{Ad}_{m}^{*} \sigma=\sigma .
$$

Так как $J^{-1}(\sigma) \subset J^{-1}\left(\sigma+\left(\mathfrak{n}+\mathfrak{m}_{\nu}\right)^{\sharp}\right) \subset J^{-1}\left(\sigma+\mathfrak{n}^{\sharp}\right)$, то диаграмма

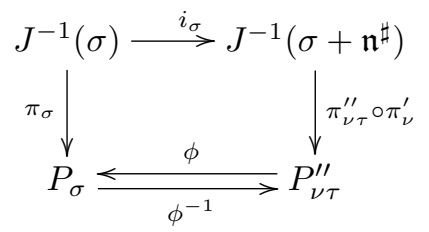

коммутативна. Отображения $\phi$ и $\phi^{-1}$ являются гладкими, поскольку отображения $\pi_{\sigma}$ и $\pi_{\nu \tau}^{\prime \prime} \circ \pi_{\nu}^{\prime} \circ i_{\sigma}$ являются субмерсиями. Отметим также, что мы не предполагаем, что подмножество $J^{-1}\left(\sigma+\left(\mathfrak{n}+\mathfrak{m}_{\nu}\right)^{\sharp}\right)$ является подмногообразием в $P$.

Теперь осталось доказать, что $\phi^{*}\left(\omega_{\sigma}\right)=\omega_{\nu \tau}^{\prime \prime}$. Чтобы это показать, отметим, что по определению симплектических форм $\omega_{\sigma}, \omega_{\nu}^{\prime}$ и $\omega_{\nu \tau}^{\prime \prime}$ для каждой точки $p \in J^{-1}(\sigma)$ и касательных векторов $Y, Z \in T_{p}\left(J^{-1}(\sigma)\right)$ имеем

$$
\begin{aligned}
\omega(Y, Z)(p) & =\omega_{\sigma}\left(\pi_{\sigma *} Y, \pi_{\sigma *} Z\right)\left(\pi_{\sigma}(p)\right) \\
\omega(Y, Z)(p) & =\omega_{\nu}^{\prime}\left(\pi_{\nu *}^{\prime} Y, \pi_{\nu *}^{\prime} Z\right)\left(\pi_{\nu}^{\prime}(p)\right) \\
& =\omega_{\nu \tau}^{\prime \prime}\left(\pi_{\nu \tau *}^{\prime \prime}\left(\pi_{\nu *}^{\prime} Y\right), \pi_{\nu \tau *}^{\prime \prime}\left(\pi_{\nu *}^{\prime} Z\right)\right)\left(\pi_{\nu \tau}^{\prime \prime}\left(\pi_{\nu *}^{\prime}(p)\right)\right) .
\end{aligned}
$$

Поскольку в силу коммутативности диаграммы (19) $\phi^{-1} \circ \pi_{\sigma}=\pi_{\nu \tau}^{\prime \prime} \circ \pi_{\nu}^{\prime}$ на подмногообразии $J^{-1}(\sigma)$, то из (20) мы получим, что $\omega_{\sigma}=\phi^{-1 *} \omega_{\nu \tau}^{\prime \prime}$. 


\section{Список литературы}

[1] J. Marsden, A. Weinstein, "Reduction of symplectic manifolds with symmetry", Rep. Mathematical Phys., 5:1 (1974), 121-130.

[2] J.E. Marsden, G. Misiołek, J.-P. Ortega, M. Perlmutter, T.S. Ratiu, Hamiltonian reduction by stages, Lecture Notes in Math., 1913, Springer-Verlag, Berlin-Heidelberg, 2007.

[3] V. Guillemin, Sh. Sternberg, "The moment map and collective motion", Ann. Physics, 127:1 (1980), 220-253.

[4] J. E. Marsden, T. Ratiu, A. Weinstein, "Semidirect products and reduction in mechanics", Trans. Amer. Math. Soc., 281:1 (1984), 147-177.

[5] J.E. Marsden, T. Ratiu, A. Weinstein, "Reduction and Hamiltonian structures on duals of semidirect product Lie algebras", Fluids and plasmas: geometry and dynamics (Boulder, CO, 1983), Contemp. Math., 28, Amer. Math. Soc., Providence, RI, 1984, $55-100$.

[6] J.E. Marsden, G. Misiołek, M. Perlmutter, T.S. Ratiu, "Symplectic reduction for semidirect products and central extensions", Differential Geom. Appl., 9:1-2 (1998), $173-212$.

[7] V. Guillemin, Sh. Sternberg, Geometric asymptotics, Mathematical surveys, 14, Amer. Math. Soc., Providence, RI, 1977; рус. пер.: В. Гийемин, С. Стернберг, Геометрические асимптотики, Мир, М., 1981.

[8] J. E. Marsden, T. S. Ratiu, Introduction to mechanics and symmetry. A basic exposition of classical mechanical systems, Texts Appl. Math., 17, Springer-Verlag, New York, 1999.

И. В. Микитюк (I. V. Mykytyuk)

Institute of mathematics, Rzesźow University, Poland;

Институт прикладных проблем механики и математики им. Я. С. Подстригача НАН Украины, г. Львов, Украина E-mail: mykytyuk_i@yahoo.com

\section{А. М. Степин (А. M. Stepin)}

Механико-математический факультет

Московского государственного университета

им. М. В. Ломоносова;

Российский государственный гуманитарный университет

E-mail: ivan07@mech.math.msu.su
Поступила в редакцию

30.12 .2007 